\title{
Comparison of Coded-wire Tagging with Parentage-based Tagging and Genetic Stock Identification in Large-scale Coho Salmon Fisheries Applications in British Columbia, Canada
}

\author{
Terry D. Beacham ${ }^{1}$, Colin Wallace ${ }^{1}$, Kim Jonsen ${ }^{1}$, Brenda McIntosh ${ }^{1}$, John R. Candy ${ }^{1}$, David Willis ${ }^{2}$, Cheryl \\ Lynch $^{2}$, Jean-Sébastien Moore ${ }^{3}$, Louis Bernatchez ${ }^{3}$, and Ruth E. Withler ${ }^{1}$ \\ ${ }^{1}$ Fisheries and Oceans Canada, Pacific Biological Station, 3190 Hammond Bay Road, Nanaimo, B.C. \\ V9T 6N7, Canada
${ }^{3}$ Département de Biologie, Institut de Biologie Intégrative et des Systèmes (IBIS), Université Laval, Québec, Québec, GIV OA6, Canada \\ ${ }^{2}$ Fisheries and Oceans Canada, Regional Headquarters, 401 Burrard Street, Vancouver, B.C., V6C 3S4, Canada
}

Keywords: British Columbia, coho salmon, genetic stock identification, parentage-based tagging, single nucleotide polymorphisms

Since the late 1990s, all coho salmon (Oncorhynchus kisutch) released from many hatcheries in southern British Columbia (BC), Washington, and Oregon have received an adipose fin clip (termed mass marking) in order to facilitate mark-selective fisheries intended to harvest hatchery salmon only, with most clipped individuals carrying no coded-wire tag (CWT). This approach has resulted in reduced exploitation of naturally-spawned coho salmon, especially in sport fisheries, but the presence of many adipose-clipped salmon without a CWT has impaired the efficiency of CWT recovery. In spite of implementation of an electronic tag detection system to pre-screen a portion of the commercial catch to identify salmon with a CWT, the processing of many heads without a CWT from voluntary recreational recoveries and the increasing costs of CWT application and recovery have caused degradation of the information obtained from the current Canadian coho salmon assessment program.

A new, cost-effective approach to the assessment and management of wild coho salmon, and the associated hatchery production and fishery management is needed. Anderson and Garza (2006) noted that parentage-based tagging (PBT) provides equivalent information (hatchery of release, age of individual) for hatchery fish as do CWTs; implementation of PBT thus may overcome problems associated with CWT-based assessment and management of coho salmon fisheries in BC. Additionally, PBT provides a means of improved hatchery broodstock management, as well as assessment of hatchery-wild interactions in salmonids. Unlike CWT-based management, PBT-informed hatchery and fishery management would benefit from the complete adipose-clipping of hatcheryproduced salmon. A significant advantage of the combination of mass marking and PBT implementation is the capability to identify visually, sample, and if desired, remove hatchery fish of local and stray origin in threatened wild populations. Moreover, PBT entails genotyping the entire hatchery broodstock and enables the identification of all hatchery progeny by parentage assignment (Anderson 2012; Wang 2016), thus enabling a 'mark rate' of virtually $100 \%$ of hatchery fish. Steele et al. (2013) demonstrated the equivalency of CWT and PBT in an initial evaluation of population and age assignment in steelhead trout (Oncorhynchus mykiss) of the Snake River basin in the Columbia River drainage. Hess et al. (2016) expanded the approach by using both PBT and genetic stock identification (GSI) to investigate run timing of steelhead trout in the upper Columbia River drainage. These applications confirmed the capability of a combined PBT-GSI technology to provide equivalent or better identification of fish as the CWT method, but were limited in geographic scale.

The study is an evaluation of the application of the PBT-GSI methodology outlined by Beacham et al. (2017) to coho salmon fisheries in $\mathrm{BC}$ to determine whether the genetic technologies can be used to provide more information on fishery contributions by hatchery and CU than is available from CWTs. Commercial and recreational coho salmon fisheries, and river escapements for selected populations, were sampled for both CWTs and genotypes. We evaluated the population-level resolution obtained from CWTs and the genetic methodology by CU for all 2017 and some 2016 fisheries in which coho salmon were caught, catch estimation by CU for the fisheries sampled, and stock-specific exploitation rate for selected populations of coho salmon in BC. Genotyping by sequencing methodology was used to genotype coho salmon at 304 single nucleotide polymorphisms (SNPs) in 304 amplicons. Complete broodstock genotyping for PBT analysis was conducted in 2014 for 20 hatcheryenhanced populations that included genotyping 6,061 individuals (96.4\% genotyping success rate), and a stock identification baseline comprising some 267 populations ranging from southeast Alaska to Oregon was employed for GSI. A comparison of the population-specific contributions to mixed-stock fisheries, catch, and exploitation rates estimated with CWTs and PBT-GSI technologies was made. We conclude that a genetic approach can emulate 
and improve upon the results available from the current CWT program for assessment and management of coho salmon enhancement and fisheries in BC, and provide critical information to improve wild coho salmon assessment and conservation.

The detailed procedure for library preparation and genotyping was outlined by Beacham et al. (2017), and a version provided by Beacham et al. (2019). Summarized briefly, 756 individuals with up to 490 amplicons per individual were loaded on a P1 chip v3 with an Ion Chef, two chips were loaded consecutively with one run of the Ion Chef, both chips were then subsequently loaded on to an Ion Torrent Proton sequencer, and the genotype of each individual was scored and recorded with Proton software Variant Caller® at multiple SNP sites in each amplicon. Genotypes at all available SNPs for an individual at the two amplicons were assembled to provide a multi-locus individual genotype, with special emphasis on those sites in the amplicons where all individuals in a species were homozygous and alternate homozygotes were present at the same site in other species.

For seven populations where CWTs were applied and the 2014 broodstock genotyped, there were 352 CWTs recovered from individuals in these seven populations sampled in Canadian fisheries. Of these individuals, 335 were sampled for potential genotyping (individuals sampled in test fisheries were not included in the samples to be genotyped), and genotypes were obtained from $86.0 \%$ (288/335) of the initial individuals processed. PBT assignments were made for $92.0 \%$ (265/288) of the genotyped individuals, and PBT assignments were $100 \%$ accurate with respect to population of origin and age in comparison with CWTs. There were 285 additional PBT assignments made for these seven populations, which were individuals that had been adipose fin clipped but were not tagged with a CWT. For the sample provided, $335 \mathrm{CWT}$ from the seven populations were recovered, and 500 PBT assignments were made, with $49 \%$ more individual identifications through PBT than with CWTs. In addition, 680 PBT assignments were made for 13 populations where no CWTs were applied, with $367 \%$ (1,230 PBT versus 335 CWT) more individual assignments made for the same base sample. A more complete description of results available from the study was outlined by Beacham et al. (2019).

Genetic-based assessment benefits from mass marking of hatchery production, particularly with regards to escapement sampling. The proportion of hatchery-origin fish in the escapement can be determined visually as the proportion of individuals missing the adipose fin, without any further sampling required. The broodstock and nonbroodstock escapement sampling in the current project generally indicated very low rates of straying among sampled populations, and thus if the escapement abundance is known or estimated, the hatchery portion of the escapement for a population can be estimated via the observed clip rate. No genotyping of non-broodstock escapement is required in order to estimate the hatchery component of the escapement. However, if survival of different release groups is required to be evaluated, escapement sampling is required to assign individuals to parents and therefore release group.

The PBT-GSI approach to fishery assessment enables catch by CU to be determined for any fishery in the province, and a means to implement the conservation/harvest balance that could be achieved by managing a combination of mixed-stock ocean fisheries and potential in-river fisheries targeting only healthy CUs (Price et al. 2017), providing substantial improvement to both CU status assessment as required by the WSP (DFO 2005) and MU fishery management. The use of PBT to identify members of hatchery or wild indicator populations and GSI to identify remaining individuals in the catch identifies the previously unknown components of the harvest when assessed with CWTs.

The strongest benefits of a PBT-GSI management system come from the additional information that it can provide, not only for improved fishery management but also for wild population conservation and management of enhancement programs. Currently, few wild populations are marked with CWTs in BC, and they are assumed to be reliable proxies for coho salmon populations over large geographic regions that may encompass multiple CUs. For the first time, analysis of northern and central coast fisheries in this study enabled comprehensive determination of fishery impacts on central/northern river systems and their constituent CUs.

This study has demonstrated the potential for implementation of a comprehensive PBT-GSI methodology for management and assessment of coho salmon in British Columbia that will remedy noted deficiencies of the current CWT-based management system. Most importantly, the genetic technology provides an immediate tool for identification of coho salmon to $\mathrm{CU}$, a requirement for implementation of management of wild populations as mandated by the WSP for Pacific salmon, and a task that would be prohibitively expensive using CWTs. Moreover, the PBT-GSI technology benefits from the mass marking of hatchery-produced salmon, thereby facilitating improved hatchery broodstock management, monitoring of wild-enhanced fish interactions, and the evaluation of hatchery contributions to harvest. The ability to identify readily hatchery-produced salmon has been recognized as an imperative for managing the risks and assessing the benefits of hatchery production of salmonids at the domestic, bilateral, and international levels (Ruggerone and Irvine 2018). In Canada, extensive coho salmon conservation and enhancement efforts conducted for two decades requires comprehensive evaluation and possible modification that 
cannot be achieved under the current management system. The genetic methodology developed in this study provides an opportunity for conservation-based management of Canadian coho salmon in which the economic benefit of hatchery production can be reaped without the imposition of undue and unknown risk to wild populations.

\section{REFERENCES}

Anderson, E.C. 2012. Large-scale parentage inference with SNPs: an efficient algorithm for statistical confidence of parent pair allocations. Stat. Appl. Genet. Mol. 11(5): 1544-6115. doi:10.1515/1544-6115.1833.

Anderson, E.C., and J.C. Garza. 2006. The power of single-nucleotide polymorphisms for large-scale parentage inference. Genetics. 172(4): 2567-2582. doi:10.1534/genetics.105.048074.

Beacham, T.D., C. Wallace, C. MacConnachie, K. Jonsen, B. McIntosh, J.R. Candy, R. Devlin, and R. E. Withler. 2017. Population and individual identification of Coho salmon in BC through parentage-based tagging and genetic stock identification: an alternative to coded-wire tags. Can. J. Fish. Aquat. Sci. 74: 1391-1410.

Beacham, T.D., C. Wallace, K. Jonsen, B. McIntosh, J.R. Candy, D. Willis, C. Lynch, J. Moore, L. Bernatchez, and R.E. Withler. 2019. Comparison of coded-wire tagging with parentage-based tagging and genetic stock identification in a large-scale coho salmon fisheries application in British Columbia, Canada. Evol. Appl. 12: 230-254. doi:10.1111/eva.12711.

Hess, J.E., M.W. Ackerman, J.K. Fryer, D.J. Hasselman, C.A. Steele, J.J. Stephenson, J.M. Wheteaker, and S.R. Narum. 2016. Differential adult migration-timing and stock-specific abundance of steelhead in mixed stock assemblages. ICES J. Mar. Sci. 73: 2606-2615. doi:10.1093/icesjms/fsw138.

Price, M.H.H., K.K. English, A.G. Rosenberger, M. MacDuffee, and J.D. Reynolds. 2017. Canada’s Wild Salmon Policy: an assessment of conservation progress in British Columbia. Can. J. Fish. Aquat. Sci. 74: 1507-1518.

Ruggerone, G.T., and J.R. Irvine. 2018. Numbers and biomass of natural- and hatchery-origin pink salmon, chum salmon, and sockeye salmon in the north Pacific Ocean, 1925-2015. Mar. Coast. Fish. 10: 152-168.

Steele, C.A., E.C. Anderson, M.W. Ackerman, M.A. Hess, N.R. Campbell, S.R. Narum, and M.R. Campbell. 2013. A validation of parentage-based tagging using hatchery steelhead in the Snake River basin. Can. J. Fish. Aquat. Sci. 70: 1046-1054.

Wang, J. 2016. Individual identification from genetic marker data: developments and accuracy comparisons of methods. Mol. Ecol. Resour. 16: 163-175. 